\title{
Insufficient evidence to understand effect of routine scaling and polishing
}

\author{
What are the benefits and harms of routine scaling and polishing for \\ periodontal health and do these change with different time intervals?
}

\begin{abstract}
Beirne P, Forgie A, Worthington HV, Clarkson JE. Routine scale and polish for periodontal health in adults (Cochrane Review). In the Cochrane Library. 2005: Issue 1, Chichester: John Wiley

Data sources Relevant studies were sourced using the Cochrane Oral Health Group Trials Register, the Cochrane Central Register of Controlled Trials, Medline and Embase. Reference lists from relevant articles were scanned and the authors of eligible studies were contacted where possible to identify trials and obtain additional information.

Study selection Trials were selected if they were randomised, included anyone with an erupted permanent dentition, and where subjects were judged to have received a routine scale and polish as

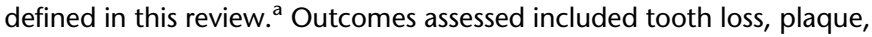
calculus, gingivitis, bleeding and periodontal indices, changes in probing depth, attachment change, patient-centred outcomes and economic outcomes.
\end{abstract}

Data extraction and synthesis Trial details were independently extracted, in duplicate, by two reviewers. Authors were contacted where possible and where deemed necessary for further details regarding study design and for data clarification. A quality assessment of all included trials was carried out. The Cochrane Collaboration's statistical guidelines were followed and both the standardised mean differences and weighted mean differences were calculated, as appropriate, using random-effects models.

Results Eight studies were included in this review and all studies were assessed as having a high risk of bias. Two split-mouth studies provided data for the comparison between scale and polish versus no scale and polish. One study, which involved people attending a recall programme following periodontal treatment, found no statistically significant differences for plaque, gingivitis and attachment loss between experimental and control units at each timepoint during the 1-year trial. The other study, of adolescents in a developing country who had high existing levels of and calculus who had not received any dental treatment for at least 5 years, reported statistically significant improvements in calculus and gingivitis (bleeding) scores between treatment and control units at 6,12 and 22 months following a single scale and polish provided at baseline to treatment units. For comparisons between routine scale and polish procedures provided at different time intervals, there were some statistically significant differences in favour of scaling and polishing carried out at more frequent intervals, that is, at 2 weeks versus 6 months; 2 weeks versus 12 months (for the outcomes plaque, gingivitis, pocket depth and attachment change); and at 3 months versus 12 months (for the outcomes plaque, calculus and gingivitis). There were no studies comparing the effects of scaling and polishing provided by dentists or professionals complementary to dentistry.

Conclusions The research evidence is not of sufficient quality to reach any conclusions regarding the beneficial and adverse effects of routine scaling and polishing for periodontal health and regarding the effects of providing this intervention at different time intervals. High-

Address for correspondence: Emma Tavender (Review Group Co-ordinator), Cochrane Oral Health Group, Manchester Dental Education Centre, School of Dentistry, University of Manchester, Higher Cambridge Street, Manchester M15 6FH, UK. quality clinical trials are required to address the basic questions posed in this review.

${ }^{\text {a }}$ Scaling and/or polishing of the crown and root surfaces of the teeth to remove local irritational factors (plaque, calculus, debris and staining), that does not involve periodontal surgery or any form of adjunctive periodontal therapy such as the use of chemotherapeutic agents or root planing.

\section{Commentary}

Seldom has a Cochrane review addressed a question with such relevance to dental practice. It is therefore disappointing, although understandable, that the review finds the available evidence to be inadequate for any conclusions to be drawn.

The review examines the evidence that underlies the selection of periodic recall intervals from the perspective of the effect on periodontal health of differing time-intervals between scaling and polishing. The review has been performed with the usual high level of competence and attention to bias-reducing procedures characteristic of virtually all Cochrane reviews of dental questions. It is thorough, careful, and complete. Of course, it carries with it the limitation inherent in most of these reviews, of including only randomised clinical trials. For this particular question, the quantity of non-randomised trials identified in the exclusion list suggests that an independent review of this more "risky" literature might be profitable. This might answer the inevitable "what if" issues associated with the exclusion criteria, and conceivably improve our general knowledge of the presence and relative strength of any treatment effect.

The abstract of the review is perhaps too terse to communicate all of the useful observations and/ or implications for practice that can be drawn from the full report. The review essentially asked two related principal questions. The first is, do scale and polish procedures lead to any difference in periodontal health compared with no scale and polish? Second, does the interval between these scale and polishing procedures make any difference?

The outcomes examined by most studies were plaque, calculus and gingivitis; three studies also reported pocket depth and attachment change. The extremely scanty evidence (two studies) for the first question is mixed, but the authors of the only study that found differences in gingivitis scores (at 6, 12 and 22 months) deemed those differences clinically irrelevant.

The evidence for the second question (four studies) is also mixed, with some but not all studies detecting differences in gingivitis, pocket depth and attachment outcomes between the shortest and longest interval studied. Here, differences in study populations (young adults versus periodontal patients) may explain the differing results.

Two other studies, where the scale and polish was carried out on a fixed schedule compared with an 'as needed' basis, which was determined by bacteriological assessment, showed no differences.

Obviously, the results do not speak with one voice. Differences are found between frequent and infrequent intervals or no scale and polish in some studies, but not others. As the review points out, even some of the statistically significant differences reported were 
seen as clinically insignificant by the study's authors. Also, the differences were identified only for comparisons involving the shortest interval and longest interval.

The thoughtful reader might receive the impression from the review that any important clinical, periodontal effects of longer intervals between scaling and polishing are difficult to detect clinically, and that the longer-term implications of many of the effects that have been identified (more plaque, calculus and gingivitis) may not be serious for the majority of subjects. Further reflection, perhaps aided by inspection of the confidence intervals for some of the comparisons, may lead the reader to the conclusion that determination of the interval between scale and polish procedures, or indeed the need for scaling and polishing at all, is driven by individual circumstances. Hence, this matter will require the provider's clinical judgment, perhaps exercised at every recall visit.

Unfortunately, the evidence for this suggested conclusion is just as weak as for any fixed interval. With the exception of two studies (which determined the need for scale and polish procedures in the experiment group based on bacteriological criteria), none of the studies included any consideration of risk factors or clinical conditions seen at recall in the determination of the recall intervals.
Thus, the reviewers' recommendations for further research might be extended to research on risk factors, also.

Practitioners are urged in this instance to obtain, read, and reflect on the full review. Scale and polish procedures are common in most practices, and practitioners who perform and schedule these should take this opportunity to examine what they believe, in the light of what is known about its effectiveness on a periodic basis. If practitioners draw the likely conclusion that there is no support for any recall regimen based on fixed intervals between scale and polishing that does not include an evaluation of clinical outcomes, then the most important implication of the review will have been successfully transferred. It will then be on their shoulders to complete the transfer into their practices.

\section{Jim Bader \\ Department of Restorative Dentistry, University of North Carolina, Chapel Hill, North Carolina, USA}

Evidence-Based Dentistry (2005) 6, 5-6.

doi:10.1038/sj.ebd.6400317 